\title{
EFFECTIVE STABILITY AROUND PERIODIC ORBITS OF THE SPATIAL RTBP
}

\author{
ÀNGEL JORBA AND JORDI VILLANUEVA \\ Departament de Matemàtica Aplicada I (ETSEIB) \\ Universitat Politècnica de Catalunya \\ Diagonal 64\%, 08028 Barcelona (Spain)
}

\section{Introduction}

In this work we study the dynamics around an elliptic periodic orbit of Hamiltonian systems. To this end we have developped an algorithm to compute a normal form (up to a finite order) around this orbit, that gives an accurate description of the dynamics close to it. If the remainder of this normal form can be bounded, it is not difficult to produce explicit bounds of the diffusion time of trajectories starting near the periodic orbit. In order to discuss the effectivity of the method, it will be explained at the same time that it is applied to a concrete example. The one used here has been the Spatial Restricted Three Body Problem (RTBP).

\subsection{DESCRIPTION OF THE MODEL PROBLEM}

Let us consider the equilateral equilibrium point $L_{5}$. It is a well known fact that the $L_{5}$ point is linearly stable for values of the mass parameter $\mu$ less than the Routh critical value $\mu_{R}=\frac{1}{2}(1-\sqrt{23 / 27})=0.03852 \ldots$ and unstable for $\mu \geq \mu_{R}$. Moreover, for any value of $\mu$, the linearized system around $L_{5}$ always contains a vertical oscillation with frequency 1 . This oscillation produces, in the complete system, a family of periodic Lyapunov orbits. In what follows, we will refer to this family as the "vertical" family of periodic orbits, and it will be parametrized by the vertical amplitude of the orbits (this parametrization is well defined for moderate values of the amplitude).

If we compute (numerically) this vertical family of periodic orbits we can see that there are some changes of stability. For instance, for some $\mu>\mu_{R}$ one can see that the vertical family of periodic orbits is unstable if the amplitude $A$ is small enough (as expected), and that they become linearly stable for sufficiently large values of $A$. ¿From now on, we will focus on one of these linearly stable orbits. The complete study of the curves (with respect to $A$ and $\mu$ ) for which there is a stability change is actually in progress ([5]).

Using KAM-like arguments one can find that this orbits are surrounded by invariant tori, and Nekhoroshev-like arguments can allow to bound the diffussion time near the orbit to prove the so-called effective stability. This second approach has been the one used in this work. 


\subsection{RELATED WORKS}

Similar studies have been done to bound the diffusion time around elliptic fixed points (see, for instance, [3], [8], [1] and [4]). There are also some works about bounding the diffusion time near maximal dimension invariant tori (see [7] and [6]), but they are much more difficult to apply to a concrete problems. A similar normal form procedure has already been used in [4] and [9].

It is also interesting the work [2], where an unified vision of the KAM and Nekhoroshev techniques is presented.

\section{The system of reference}

The main difficulty is to obtain a system of coordinates adapted to the periodic orbit.

\subsection{FIRST STEP}

Let $\left(X, Y, Z, P_{X}, P_{Y}, P_{Z}\right)$ be a system of reference for the RTBP, centered in the equilibrium point $L_{5}$. Let us assume that we know a $2 \pi$-periodic parametrization of an orbit of the vertical family $(f(\theta), g(\theta))$ of period $2 \pi / \omega$, where $f=\left(f_{1}, f_{2}, f_{3}\right), g=\left(g_{1}, g_{2}, g_{3}\right)$ and $f_{3}(0)=0$. We suppose that the system of reference is such that the projection of the orbit into the coordinates $\left(Z, P_{Z}\right)$ is close to a circle, ${ }^{1}$ that is, we assume that $\Delta=\left(f_{3}^{\prime}\right)^{2}+\left(g_{3}^{\prime}\right)^{2}$ is close to constant (the vertical family of periodic orbits satisfies this condition).

Now, we want to introduce a new system of coordinates $\left(\theta, q_{1}, q_{2}, I, p_{1}, p_{2}\right)$ (with a canonic change of variables) to describe the periodic orbit as a function of $\theta$ with $q_{1}=q_{2}=$ $I=p_{1}=p_{2}=0$. We use the change

$$
\begin{aligned}
X & =f_{1}(\theta)+q_{1}, & P_{X} & =g_{1}(\theta)+p_{1}, \\
Y & =f_{2}(\theta)+q_{2}, & P_{Y} & =g_{2}(\theta)+p_{2}, \\
Z & =f_{3}(\theta)-\frac{g_{3}^{\prime}(\theta)}{\Delta(\theta)} \alpha(\theta, F(q, \theta, p, I)), & P_{Z} & =g_{3}(\theta)+\frac{f_{3}^{\prime}(\theta)}{\Delta(\theta)} \alpha(\theta, F(q, \theta, p, I)),
\end{aligned}
$$

with $F(q, \theta, p, I)=I+\sum_{j=1}^{2}\left(g_{j}^{\prime}(\theta) q_{j}-f_{j}^{\prime}(\theta) p_{j}\right), \alpha(\theta, s)$ verifies $s=\alpha(\theta, s)+\frac{1}{2} \sigma(\theta) \alpha(\theta, s)^{2}$ and

$$
\sigma(\theta)=\frac{g_{3}^{\prime}(\theta) f_{3}^{\prime \prime}(\theta)-g_{3}^{\prime \prime}(\theta) f_{3}^{\prime}(\theta)}{\Delta(\theta)^{2}}
$$

being $\alpha(\theta, 0)=0$. It is not dificult to check that this is a canonic transformation in some (complex) domain for the variables $(\theta, q, I, p)$.

\subsection{HAMILTONIAN IN THE NEW VARIABLES}

After this transformation the Hamiltonian takes the form:

$$
H(\theta, q, I, p)=h_{0}+\omega I+\frac{1}{2}\left(q^{t}, p^{t}\right) A(\theta)\left(\begin{array}{c}
q \\
p
\end{array}\right)+\cdots
$$

where $h_{0}$ is the energy level of the periodic orbit and $A(\theta)$ is a symmetric $2 \pi$-periodic matrix in $\theta$. The next step is to remove the angular dependence of $\theta$ in $A$.

\footnotetext{
${ }^{1}$ This assumption is only used to simplify the computation of the change of variables and to bound the region where it is well defined. The same methodology can be used when this assumption does not hold, but the details are more difficult.
} 


\subsection{FLOQUET TRANSFORMATION AND COMPLEXIFICATION}

We want to make a canonic change ( $2 \pi$-periodic in $\theta$ ) to transfom $A$ to constant coefficients. To describe the process, let us denote by $\mathcal{F}(q, p, \theta, I)$ the change of variables (1) and let $\psi(t)$ be the variational matrix, $\psi(0)=I d$, around the periodic orbit. We have that

$$
\phi(t)=(D \mathcal{F}(0,0, \omega t, 0))^{-1} \psi(t) D \mathcal{F}(0,0,0,0)
$$

is the variational matrix for the orbit in variables $(q, p, \theta, I)$. Let $\widetilde{\phi}(t)$ be the 4-dimensional matrix obtained by taking the first 4 rows and colums of $\phi(t)$ (they correspond to the variational flow in the normal directions of the periodic orbit). Let $C$ be a symplectic matrix that transforms $\tilde{\phi}(2 \pi / \omega)$ in real normal form, and let $M$ be a matrix such that $e^{M}=\tilde{\phi}(2 \pi / \omega)$ (it can be checked that, in this case, $M$ can be taken real). Then, the (canonic) change of variables

$$
\left(\begin{array}{c}
q \\
p
\end{array}\right)=B(\theta)^{-1}\left(\begin{array}{c}
x \\
y
\end{array}\right), \quad I=\xi-\frac{1}{2}\left(x^{t}, y^{t}\right) J B^{\prime}(\theta) B(\theta)^{-1}\left(\begin{array}{c}
x \\
y
\end{array}\right),
$$

where $J$ is the canonic form of $\mathbb{R}^{4}$, and $B(\theta)=C^{-1} \exp \left(\frac{1}{2 \pi} M \theta\right) \tilde{\phi}(\theta / \omega)^{-1}$ transforms the Hamiltonian into

$$
H(\theta, x, \xi, y)=h_{0}+\omega \xi+\frac{\omega_{1}}{2}\left(x_{1}^{2}+y_{1}^{2}\right)+\frac{\omega_{2}}{2}\left(x_{2}^{2}+y_{2}^{2}\right)+\cdots
$$

If we introduce new (complex) variables as $x_{j}=\left(Q_{j}+i P_{j}\right) / \sqrt{2}$ and $y_{j}=\left(i Q_{j}+P_{j}\right) / \sqrt{2}$, $j=1,2$, we obtain

$$
H(\theta, P, \xi, Q)=h_{0}+\omega \xi+i \omega_{1} Q_{1} P_{1}+i \omega_{2} Q_{2} P_{2}+\cdots
$$

This is the basic expression of the Hamiltonian that we use to perform the normal form.

\subsection{EXPANSION OF THE HAMILTONIAN}

Our purpose now is to perform these changes of variables in the initial Hamiltonian. The Hamiltonian of the RTBP can we written, in suitable coordinates, as

$$
H\left(x, y, z, p_{x}, p_{y}, p_{z}\right)=\frac{1}{2}\left(p_{x}^{2}+p_{y}^{2}+p_{z}^{2}\right)+y p_{x}-x p_{y}-\frac{1-\mu}{r_{1}}-\frac{\mu}{r_{2}},
$$

where $r_{1}^{2}=(x-\mu)^{2}+y^{2}+z^{2}$ and $r_{2}^{2}=(x-\mu+1)^{2}+y^{2}+z^{2} . L_{5}$ is located at $\left(-\frac{1}{2}+\right.$ $\left.\mu, \frac{\sqrt{3}}{2}, 0,-\frac{\sqrt{3}}{2},-\frac{1}{2}+\mu, 0\right)$. Let $\left(X, Y, Z, P_{X}, P_{Y}, P_{Z}\right)$ be the initial coordinates centered in $L_{5}$ (by means of a translation of origin), and let $(f(\theta), g(\theta))$ be the orbit in this coordinates. We assume that $\left(f_{3}^{\prime}\right)^{2}+\left(\frac{g_{3}^{\prime}}{\omega}\right)^{2}$ is close to constant, and we introduce new coordinates $\zeta=\sqrt{\omega} Z$ and $P_{\zeta}=\frac{P_{Z}}{\sqrt{\omega}}$. We rewrite the Hamiltonian in this coordinates but we keep for simplicity the notation $Z, P_{Z}$. Then, we expand the Hamiltonian as

$$
\begin{aligned}
H=\frac{1}{2} & \left(P_{X}^{2}+P_{Y}^{2}+\omega P_{Z}^{2}\right)+Y P_{X}-X P_{Y}+\frac{X^{2}}{8}-\frac{5}{8} Y^{2}-a X Y+ \\
& +\frac{1}{2} \frac{Z^{2}}{\omega}-\sum_{k \geq 3} r_{0}^{k} P_{k}\left(\frac{X-\sqrt{3} Y}{2 r_{0}}\right)(1-\mu)-\sum_{k \geq 3} r_{0}^{k} P_{k}\left(\frac{-X-\sqrt{3} Y}{2 r_{0}}\right) \mu,
\end{aligned}
$$


being $a=-\frac{3 \sqrt{3}}{4}(1-2 \mu), r_{0}^{2}=X^{2}+Y^{2}+\frac{Z^{2}}{\omega}$ and $P_{k}$ the Legendre polynomial of degree $k$. To expand the Hamiltonian we compute the composition of the changes (1) and (2) and we use the recurrence of the Legendre polynomials.

\section{Computing the normal form}

To simplify the notation, let us rename $Q_{i}$ as $q_{i}, P_{i}$ as $p_{i}$ and $\xi$ as $I$. With this, we have obtained a Hamiltonian of the form:

$$
H(\theta, q, I, p)=\omega I+i \omega_{1} q_{1} p_{1}+i \omega_{2} q_{2} p_{2}+H_{2}(\theta, q, I, p)+H_{3}(\theta, q, I, p)+\cdots,
$$

where $H_{j} j \geq 3$ contains monomials in $q, p$ and $I$ of degree $j$, with periodic coefficients in $\theta$, and all the monomials of $H_{2}$ contain the variable $I$ (and are of degree 2). Our objective is to put the Hamiltonian in normal form up to some finite order by using a canonic change $(2 \pi$ periodic in $\theta$ ) of variables that we obtain as a composition of time unity flows associated to Hamiltonians (generating functions) $G_{j}$. They are selected to remove, in recursive form, the nonintegrable terms of degree $j$. So, if we have $G_{2}, G_{3}, \ldots, G_{n}$ that

$$
H \circ \Phi_{G_{2}}^{t=1} \circ \cdots \circ \Phi_{G_{n}}^{t=1}(\theta, q, I, p)=\omega I+i \omega_{1} q_{1} p_{1}+i \omega_{2} q_{2} p_{2}+N^{(n)}+H_{n+1}^{(n)}+H_{n+2}^{(n)}+\cdots,
$$

where $N^{(n)}$ is in normal form up to degree $n$. We take $G_{n+1}$ to put $H_{(n+1)}^{(n)}$ in normal form, that is, we select $G_{n+1}$ such that $\left\{\omega I+i \omega_{1} q_{1} p_{1}+i \omega_{2} q_{2} p_{2}, G_{n+1}\right\}+H_{n+1}^{(n)}$, has only exact resonant terms, where

$$
\{f, g\}=\frac{\partial f}{\partial \theta} \frac{\partial g}{\partial I}-\frac{\partial f}{\partial I} \frac{\partial g}{\partial \theta}+\sum_{j=1}^{2}\left(\frac{\partial f}{\partial q_{j}} \frac{\partial g}{\partial p_{j}}-\frac{\partial f}{\partial p_{j}} \frac{\partial g}{\partial q_{j}}\right)
$$

is the Poisson bracket. More details of this process can be found in [4] or [9].

\subsection{REMARK}

In fact the good order to remove the diferent terms is not the order that give the usual definition of degree of homogeneous polynomials, but the one defined counting twice the degree of the $I$ variable. This allows us to remove the non integrable part of $H_{2}$.

\section{Normal form}

Finally we have a Hamiltonian like $H(\theta, q, I, p)=N\left(I, q_{1} p_{1}, q_{2} p_{2}\right)+R(\theta, q, I, p)$ where $H$ is in normal form up to degree $n$. It is easy to see that the construction of the normal form keeps the real character of the Hamiltonian, and consequently we can introduce new real variables to describe the real behaviour of the orbit.

\section{Some results}

We have applied the latter procedure taking $\mu=0.04$ (that is, bigger than the Routh critical value), and we have used the Lyapunov orbit that has $\dot{z}=0.2474290163$ when $z=0$, in the initial coordinates. This orbit is linearly stable.

The software used has been specially developed, in C language, for this problem. This allows to improve, in several orders of magnitude, the efficiency (both in speed and memory) obtained by using commercial algebraic manipulators. 


\subsection{INVARIANT TORI}

Now it is easy to obtain aproximations of periodic orbits and invariant tori of dimension 2 and 3 of the Hamiltonian in normal form if we ignore the remainder $R$. If we transform them for all the changes we can obtain aproximations of periodic orbits and tori of the initial system.

\subsection{EFFECTIVE STABILITY}

To obtain estimates for the region where there is long time stability, we need to bound the remainder $R$. With this we can estimate the effective stability domain in the normal form variables. The next step is to obtain information about how is this domain is mapped onto the initial variables by the succesive changes of variables.

To perform this bounds, we need to obtain estimates of the norms of the remaining terms that we can not store in a practical implementation of the method. This has been done numerically, and the results show a (quite small) neighbourhood of practical stability around the orbit. This is because this orbit is very close to the bifurcation that changes the stability of the vertical family. Full details can be found in [5].

\section{Acknowledgements}

A. Jorba has been partially supported by the grants DGICYT PB94-0215, CIRIT GRQ931135, the EC grant ERBCHRXCT940460 and the UPC fund PR9409. J. Villanueva has been supported by the UPC fund PR9409.

\section{References}

1. Celleti, A. and Giorgilli, A. (1991) On the stability of the Lagrangian points in the spatial restricted problem of three bodies, Cel. Mech., 50, pp. 31-58.

2. Delshams, A. and Gutiérrez, P. (1995) Effective stability and KAM Theorem, to appear in $J$. of Diff. Equations.

3. Giorgilli, A., Delshams, A., Fontich, E., Galgani, L. and Simó, C. (1989) Effective stability for a Hamiltonian system near an elliptic equilibrium point, with an application to the Restricted Three Body Problem, J. of Diff. Equations, 77:1, pp. 167-198.

4. Jorba, A. and Simó, C. (1994) Effective stability for periodically perturbed Hamiltonian systems, in Hamiltonian Mechanics, Integrability and Chaotic Behavior, J. Seimenis (Ed.), Plenum Press, New York, pp. 245-252.

5. Jorba, A. and Villanueva, J. (1995) Normal form around periodic orbits of the spatial RTBP, in progress.

6. Morbidelli, A. and Giorgilli A. (1995) Superexponential stability of KAM tori, J. Stat. Phys. 78, no. $5 / 6$.

7. Perry, A. D. and Wiggins, S. (1994) KAM tori are very sticky: rigorous lower bounds on the time to move away from an invariant Lagrangian torus with linear flow, Physica D, 71, pp. 102-121.

8. Simó, C. (1989) Estabilitat de sistemes Hamiltonians, Mem. de la Real Acad. de Ciencias y Artes de Barcelona, Vol. XLVIII, no. 7, pp. 303-348.

9. Simó, C., Gómez, G., Jorba, A. and Masdemont, J. (1995) The bicircular model near the triangular libration points of the RTBP, in From Newton to Chaos, A. E. Roy and B. A. Steves (Eds.), Plenum Press, New York, pp. 343-370. 